\title{
Postoperative Cognitive Decline
}

\author{
Phillip Gordon* \\ Department of Anesthesiology, California Pacific Medical Center, USA
}

*Corresponding author: Phillip Gordon, Department of Anesthesiology, California Pacific Medical Center, PO Box 7999, San Francisco, CA 94120, USA

To Cite This Article: Phillip Gordon. Postoperative Cognitive Decline. Am J Biomed Sci \& Res. 2019 - 4(4). AJBSR.MS.ID.000806. DOI: 10.34297/ AJBSR.2019.04.000806

Received: July 23, 2019 | Published: August 06, 2019

\section{Opinion}

Post-operative cognitive decline (POCD), described many years ago [1], remains a challenge for all physicians who care for elderly patients. A lot of work has been done in this area but investigators need to be mindful about incorporating some basic precepts into their research protocols. While central nervous system dysfunction after surgery and anesthesia has been described to occur, especially in older adults, it is important to bear in mind that, as Silverstein and colleagues [2] point out, any illness requiring hospitalization may be associated with cognitive decline. This introduces the possibility that cognitive decline occurs as a concomitant of generalized illness rather than being causally related to surgery and anaesthesia and speaks to the need for not only age-matched controls but also disease-matched and hospitalization-matched clinical cohorts, as well. Another confounder of the correlation between POCD and mortality reported by Monk and colleagues [3] is the observation made by a number of investigators that cognitive decline itself increases the risk of mortality in older adults $[4,5]$. As suggested by Newman and colleagues [6], the very term POCD as a binary definition of what may be, in reality, a continuous process may require modification to reflect the necessity of examining cognitive change as a continuum that marches through discrete events such as surgery and anesthesia. This will necessarily influence the methodology by which changes in test performance are analyzed.

Of major but little-recognized importance is the 15-minute neurological examination developed by Inzitari and colleagues [7] to elicit subtle but clinically detectable neurological abnormalities (SNAs). These SNAs are defined as abnormalities demonstrated in the absence of patient complaints which are also not associated with any neurological disease. Follow-up examination at four and eight years revealed that patients who had three or more SNAs at initial evaluation had an increased risk of cognitive decline, cerebrovascular events, and death, possibly because the SNAs signaled a concomitant decline in cognitive reserve [8]. The authors did not track whether patients had had surgery and anesthesia during the follow-up period. In light of the progression of the otherwise non-detected neurological deterioration demonstrated by Inzitari and colleagues [7], it is therefore essential that studies of POCD include a uniform neurological examination of all patients and control subjects. The neurological examination must have good inter-rater agreement and reproducibility. Without prior knowledge of SNAs, it is impossible to assign causation to the cognitive dysfunction that may occur after surgery and anesthesia. Is it the normal progression of a condition, however subtle, present preoperatively or is it the effect of surgery and anesthesia alone or have surgery and anesthesia worked in some way to hasten the progression of the preoperatively present condition? Clearly, whatever else the experimental protocols include to achieve uniformity and facilitate inter-study comparison (age- and disease-matched controls in every study and uniformity of operation, anesthesia, neuropsychological evaluation tools, testing intervals, statistical analysis, control subjects), neurological examination for both gross and subtle neurological abnormalities must be included too.

Even in the absence of POCD study protocols, preoperative information about SNAs would facilitate the discussion of informed consent when older adults are contemplating surgical procedures as neurological abnormalities, even in the absence of overt symptoms, have been correlated with POCD [3]. It may thus be advisable for an SNA "score," as developed by Inzitari and colleagues [7] with their brief neurological examination, to be included with the other vital signs measured in older adults before surgery. Clinicians can work to mitigate some of the risk factors associated with POCD. For example, postoperative delirium [9], a correlate of POCD [10], is associated with increased intraoperative blood loss and postoperative transfusions, a postoperative hematocrit of less than $30 \%$, and severe postoperative pain. The Hospital Elder Life Program has also developed interventions to decrease the incidence and severity of delirium. These include the frequent presentation of orienting information, physical activity, cognitive stimulation activities, use of visual aids and auditory amplifying devices, sleep inducement 
through non-pharmacological methods, assistance with alimentation, geriatric-psychiatric consultations, and patient and family education. The risk of delirium has been shown to be reduced using these techniques $[11,12]$ and thus perhaps the incidence of POCD.

\section{References}

1. Bedford PD (1955) Adverse cerebral effects of anaesthesia on old people. Lancet 269(6884): 259-263.

2. Silverstein JH, Timberger M, Reich DL, Uysal S (2007) Central nervous system dysfunction after noncardiac surgery and anesthesia in the elderly. Anesthesiol 106(3): 622-628.

3. Monk TG, Weldon BC, Garvan CW, Dede DE, van der Aa MT, et al. (2008) Predictors of cognitive dysfunction after major noncardiac surgery. Anesthesiol 108(1): 18-30.

4. Schupf N, Tang MX, Albert SM, Costa R, Andrews H, et al. (2005) Decline in cognitive and functional skills increases mortality risk in nondemented elderly. Neurology 65(8): 1218-1226.

5. Van Gelder BM, Tijhuis MA, Kalmijn S, Giampaoli S, Kromhout D (2007) Decline in cognitive functioning is associated with a higher mortality risk. Neuroepidemiology 28(2): 93-100.
6. Newman S, Stygall J, Shashivadan H, Shaefi S, Maze M (2007) Postoperative cognitive dysfunction after noncardiac surgery: A systematic review. Anesthesiol 106(3): 572-590.

7. Inzitari M, Pozzi C, Ferrucci L, Chiarantini D, Rinaldi LA, et al. (2008) Subtle neurological abnormalities as risk factors for cognitive and functional decline, cerebrovascular events, and mortality in older community-dwelling adults. Arch Intern Med 168(12): 1270-1276.

8. Stern Y (2002) What is cognitive reserve? Theory and research application of the reserve concept. J Int Neuropsychol Soc 8(3): 448-460.

9. Fong HK, Sands LP, Leung JM (2006) The role of postoperative analgesia in delirium and cognitive decline in elderly patients: A systematic review. Anesth Analg 102(4): 1255-1266.

10. Rudolph JL, Marcantonio ER, Culley DJ, Silverstein JH, Rasmussen LS, et al. (2008) Delirium is associated with early postoperative cognitive dysfunction. Anaesthesia 63(9): 941-947.

11. Inouye SK (2006) Delirium in older persons. N Engl J Med 354: 11571165.

12. Marcantonio ER, Flacker JM, Wright RJ, Resnick NM (2001) Reducing delirium after hip fracture: A randomized trial. J Am Geriatr Soc 49(5): 516-522. 